UCRL-JC-121488 Rev 2

PREPRINT

\title{
Supernova-Relevant Hydrodynamic Instability Experiments on the Nova Laser
}

\author{
J. Kane, D. Arnett, B. A. Remington, \\ S. G. Glendinning, R. Wallace, R. Managan, \\ A. Rubenchik, and B. A. Fryxell
}

This paper was prepared for submittal to the

Thirteenth International Conference on Laser Interactions

and Related Plasma Phenomena

Monterey, California

April 13-18, 1997

April 18, 1997

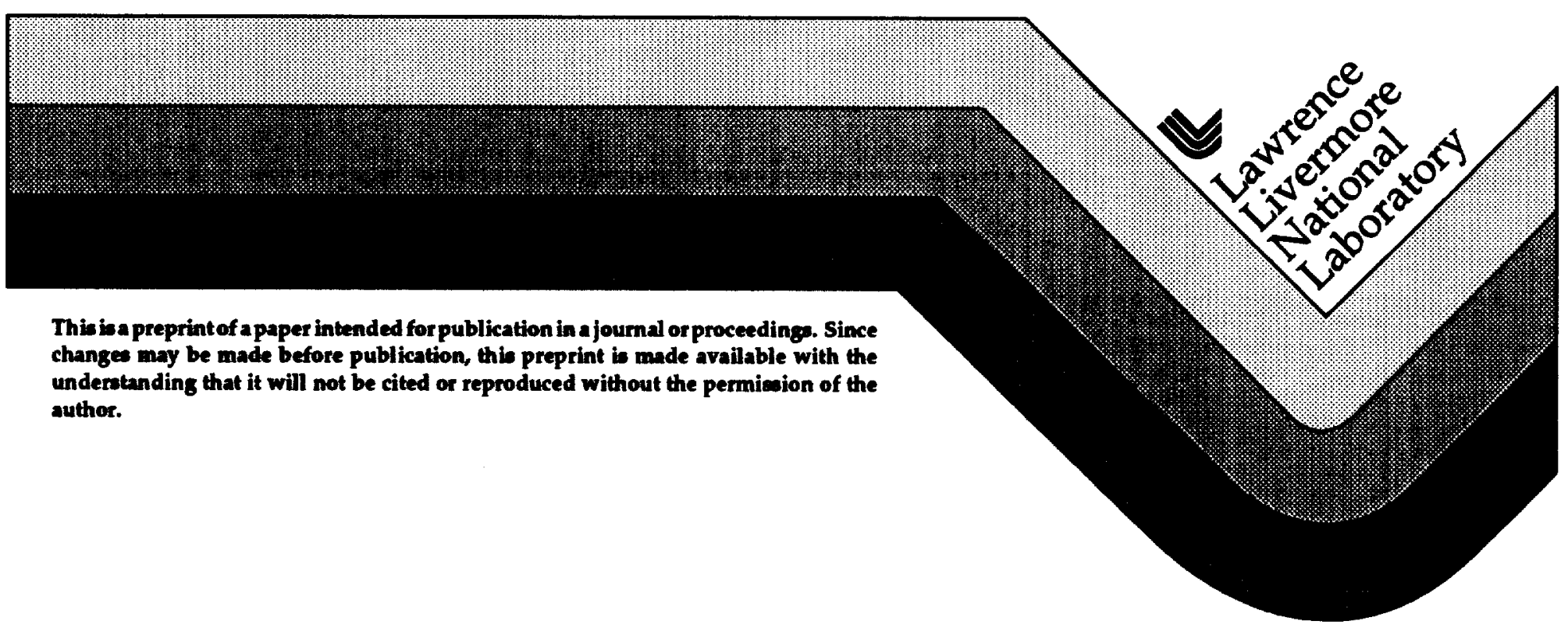




\section{DISCLAIMER}

This document was prepared as an account of work sponsored by an agency of the United States Government. Neither the United States Government nor the University of California nor any of their employees, makes any warranty, express or implied, or assumes any legal liability or responsibility for the accuracy, completeness, or usefulness of any information, apparatus, product, or process

disclosed, or represents that its use would not infringe privately owned rights. Reference herein to any specific commercial product, process, or service by trade name, trademark, manufacturer, or otherwise, does not necessarily constitute or imply its endorsement, recommendation, or favoring by the United States Government or the University of California. The views and opinions of authors expressed herein do not necessarily state or reflect those of the United States Government or the University of California, and shall not be used for advertising or product endorsement purposes. 


\title{
Supernova-relevant hydrodynamic instability experiments on the Nova laser
}

\author{
J. Kane*t and D. Arnett* \\ B. A. Remington ${ }^{\dagger}$, S. G. Glendinning ${ }^{\dagger}$, R. Wallace $^{\dagger}$, \\ R. Managan ${ }^{\dagger}$, A. Rubenchik ${ }^{\dagger \dagger}$, B. A. Fryxell ${ }^{1} 1$ \\ * University of Arizona, Tucson, AZ 85721 \\ ${ }^{\dagger}$ Lawrence Livermore National Laboratory (LLNL), Livermore CA 94550 \\ $\$$ University of California Davis \\ "Institute for Computational Science and Informatics, George Mason University, Fairfax, \\ VI 22030
}

\begin{abstract}
Supernova 1987A focused attention on the critical role of hydrodynamic instabilities in the evolution of supernovae. To test the modeling of these instabilities, we are developing laboratory experiments of hydrodynamic mixing under conditions relevant to supernovae. The target consists of a twolayer planar package composed of $85 \mu \mathrm{m} \mathrm{Cu}$ backed by $500 \mu \mathrm{m} \mathrm{CH}$, having a single mode sinusoidal perturbation at the interface, with $\lambda=200 \mu \mathrm{m}, \eta_{0}=20$ $\mu \mathrm{m}$. The Nova laser is used to generate a $10-15 \mathrm{Mbar}\left(10-15 \times 10^{12}\right.$ dynes $\left./ \mathrm{cm}^{2}\right)$ shock at the interface, which triggers perturbation growth, due to the RichtmyerMeshkov instability followed by the Rayleigh-Taylor instability as the interface decelerates. This resembles the hydrodynamics of the He-H interface of a Type II supernova at intermediate times, up to a few $\times 10^{3} \mathrm{~s}$. The experiment is modeled using the hydrodynamics codes HYADES and CALE, and the supernova code PROMETHEUS. We are designing experiments to test the differences in the growth of $2 \mathrm{D}$ vs. 3D single mode perturbations; such differences may help explain the high observed velocities of radioactive core material in SN1987A. Results of the experiments and simulations are presented.
\end{abstract}

\section{INTRODUCTION}

Observations of SN1987A, a core collapse supernova (SN) in the Large Magellanic Cloud, strongly suggested the occurence of material mixing driven by the Rayleigh-Taylor (RT) instability $[26,30,5]$. Radioactive ${ }^{56} \mathrm{Co}$ from the explosively burned oxygen layer was observed much sooner after the explosion

1) Supported by the NASA ESS HPCC Program under Grant Number NAG5-2652 
than predicted by spherically symmetric explosion models, implying that the ${ }^{56} \mathrm{Co}$ had been mixed well into the outer layers. Doppler broadening of the gamma-ray and optical lines from ${ }^{56} \mathrm{Co}$ implied velocities in excess of 3000 $\mathrm{km} / \mathrm{s}[32,31,22]$, whereas modeling to date predicts maximum velocities of $\leq$ $2000 \mathrm{~km} / \mathrm{s}$. Also, the 'Bochum event' $[29,17,8]$ showed spectroscopic features suggesting enhanced heating of the envelope, presumably due to mixing from the radioactive core.

Given the fundamental role played by the RT instability in SN evolution, it is desirable to develop the means of testing the hydrodynamics of the SN codes. We report here on first results of an experiment using the Nova laser at Lawrence Livermore National Laboratory (LLNL) to test the modeling of compressible RT instabilities at relevant pressures. We use the SN code PROMETHEUS to model the experiment, and for comparison, the LLNL code CALE.

\section{1D SN SIMULATIONS}
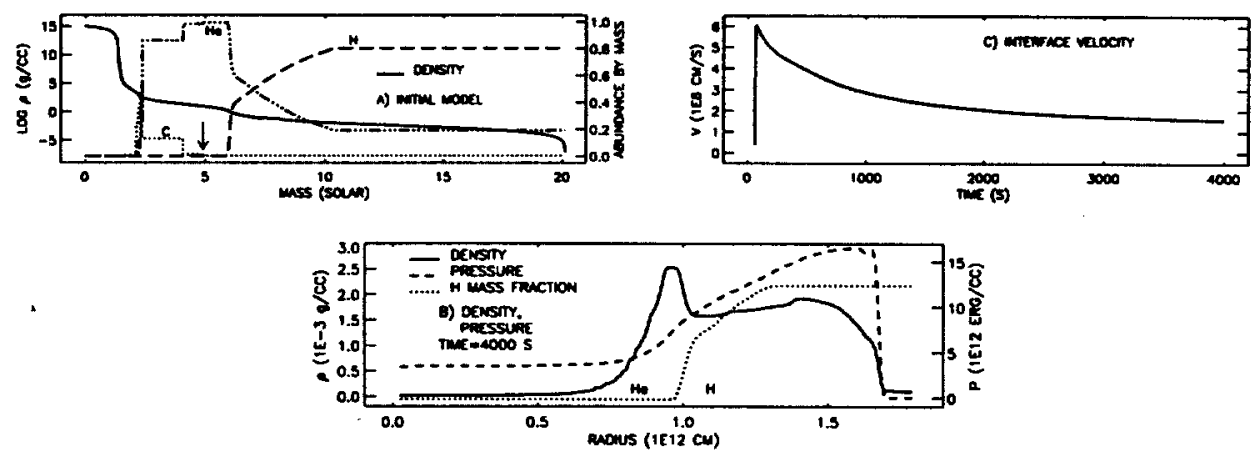

FIGURE 1. PROMETHEUS 1D SN simulation. a) The heavy line is density as a function of mass coordinate, and the other lines show the mass fractions of $\mathrm{C}, \mathrm{He}$, and $\mathrm{H}$. The arrow indicates the inner radius of our simulation, at $\approx 5 \mathrm{M}_{\odot}$, where the mass fraction of He is maximum and the $\mathrm{C}$ mass fraction is negligible. b) Pressure and density versus radius at $4000 \mathrm{~s}$. The dashed curve is the mass fraction of $\mathrm{H}$, ranging from zero for $R \lessgtr 0.9 \times 10^{12} \mathrm{~cm}$, to 0.8 for $R Z 1.3 \times 10^{12} \mathrm{~cm}$, with the He-H 'interface' occurring at $R \approx 1.0 \times 10^{12} \mathrm{~cm}$. We do not simulate the core collapse or the passage of the shock through the lower layers; we simply deposit the explosion energy $\left(1.5 \times 10^{51}\right.$ ergs) as half thermal and half kinetic energy (the later results are insensitive to the exact ratio) at the radius shown by the arrow in a). We replace the 'core' (below the arrow,) by a reflecting boundary. The equation of state (EOS) used here is fully ionized ideal gas plus radiation pressure. c) $\mathrm{He}-\mathrm{H}$ interface velocity versus time.

Fig. 1a shows a $20 \mathrm{M}_{\odot}$ model for the progenitor of SN 1987A [4]. After core collapse, a strong shock moves radially out through the star, providing the velocity impulse to the He layer less than a minute after collapse [5]. The one dimensional (1D) hydrodynamics of the SN are shown in Figs. 1b and c, 
with results from a 1D PROMETHEUS calculation of the $\mathrm{He}$ and $\mathrm{H}$ layers. PROMETHEUS is a multi-dimensional Eulerian code based upon the PPM method [33]. Fig. 1b shows the density and pressure profiles of the exploding star at $4000 \mathrm{~s}$. At this point, the shock has traveled almost halfway out of the star. Fig. 1c shows the velocity of the He-H 'interface' as a function of time; the arrival of the shock at the inner radius of the He layer defines time $t=0$. At the He-H interface, the pressure and density gradients are crossed (Fig. 1b), that is, $\nabla P \cdot \nabla \rho<0$, so the He layer is decelerated by the outer, lower density $\mathrm{H}$ layer. This configuration of a heavy He layer decelerated by a low density $\mathrm{H}$ layer is expected to exhibit strong $\mathrm{RT}$ instability, with the interface evolving well into the nonlinear regime $[6,13,24]$.

\section{LASER EXPERIMENT AND 1D SIMULATIONS}
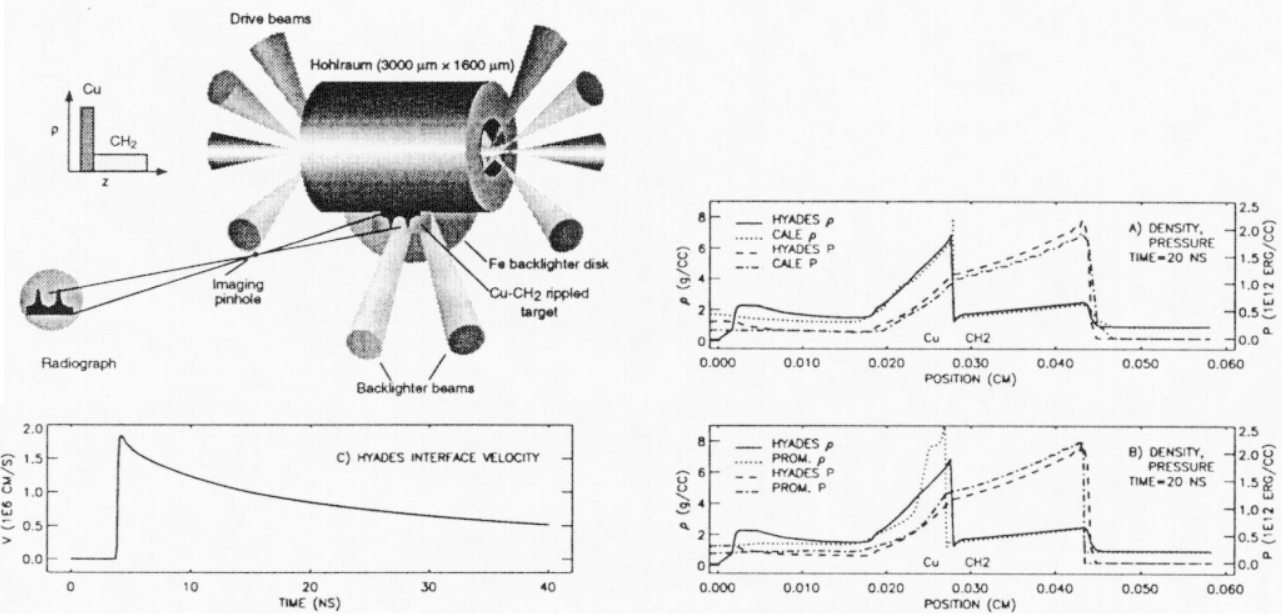

FIGURE 2. Experimental Configuration and 1D Simulations. The HYADES and the CALE simulations used a tabular equation of state for the materials, while the PROMETHEUS simulation used an ideal gas equation of state. Despite this oversimplification, the results are similar. The largest difference is the density enhancement in b).

The experimental configuration is illustrated in Fig. 2. For extensive discussions of the experimental techniques see $[25,12,9,21,19]$, and see [10] for discussion of the framing camera. Eight of the ten Nova laser beams at a wavelength of $\lambda_{L}=0.351 \mu \mathrm{m}$, energy of $1.5 \mathrm{~kJ} / \mathrm{beam}$, and duration of $1 \mathrm{~ns}$ are focused into a $3.0 \mathrm{~mm}$ long, $1.6 \mathrm{~mm}$ diameter Au hohlraum (cylindrical radiation cavity,) converting to an $\sim 190 \mathrm{ev}$, approximately thermal x-ray drive. The experimental package is planar: a $85 \mu \mathrm{m}$ thick $\mathrm{Cu}(\rho=8.9 \mathrm{~g} / \mathrm{cm})$ foil backed by $500 \mu \mathrm{m}$ of $\mathrm{CH}_{2}(\rho=0.95 \mathrm{~g} / \mathrm{cm})$. A sinusoidal ripple, of wavelength $\lambda=200 \mu \mathrm{m}$, and amplitude $\eta_{0}=20 \mu \mathrm{m}$, is imposed at this embedded interface. The package is mounted across a $750 \mu \mathrm{m}$ diameter hole in the hohlraum 
wall, so that the inner, smooth side of the $\mathrm{Cu}$ sees the x-ray drive. Diagnosis of the interface is by side-on radiography. Two Nova beams at $\lambda_{L}=0.528 \mu \mathrm{m}$, energy of $3 \mathrm{~kJ}$, and duration of $5 \mathrm{~ns}$ are focused onto a backlighter disk, a separate disk of $\mathrm{Fe}$, which generates $\mathrm{He}-\alpha \mathrm{x}$-rays at $6.7 \mathrm{keV}$. The foil, illuminated by the backlighter source, is then viewed along the length of the ripples by a gated $\mathrm{x}$-ray framing camera, so that the opaque $\mathrm{Cu}$ appears as a shadow, and the $\mathrm{CH}_{2}$ is essentially transparent.

We model the laser experiment using a combination of codes: HYADES, CALE and PROMETHEUS. The HYADES code [20] is a 1D Lagrangian code with multigroup radiation transport and tabular EOS, and CALE is a 2D Arbitrary Lagrangian Eulerian (ALE) code [7] with tabular EOS and interface tracking. PROMETHEUS was described above. Ideal gas EOS is used for all the PROMETHEUS simulations of the laser experiments. We use the measured radiation temperature, $T_{r}(t)$, as the energy input to HYADES, and the versions of CALE and PROMETHEUS that we are using do not have radiation transport.

In Figs. 2a and $\mathrm{b}$ we illustrate the results at $20 \mathrm{~ns}$ for density and pressure versus position for the simulated experiment, for all three codes. For the CALE and PROMETHEUS calculations, we map the results from HYADES as the starting conditions at $2.45 \mathrm{~ns}$. By design, the laser drive turns off at $1 \mathrm{~ns}$ and the $\mathrm{x}$-ray drive is rapidly diminishing by $2.45 \mathrm{~ns}$. Hence, the hydrodynamics at the $\mathrm{Cu}-\mathrm{CH}_{2}$ interface $85 \mu \mathrm{m}$ in from the ablation front is decoupled from the radiation drive. This mapping works well, as is illustrated in Figs. 2a and b by comparing the full 20 ns HYADES profiles for $P$ and $\rho$ with those from CALE and PROMETHEUS. Figure 2c shows the velocity of the $\mathrm{Cu}-\mathrm{CH}_{2}$ interface as simulated in HYADES. Note the impulsive shock acceleration, followed by a protracted deceleration, qualitatively similar to the $\mathrm{He}-\mathrm{H}$ interface in the SN (see Fig. 1c).

Consider the different scales of the SN and the Nova experiment, assuming the RT instability to dominate the mixing. In the nonlinear regime, the fluid flow can be characterized by a spatial scale of order the perturbation wavelength $\lambda$ and velocity of order the perturbation terminal bubble velocity $v_{\mathrm{b}} \propto \sqrt{g \lambda}$, where $g$ corresponds to the acceleration and assuming constant Atwood number $A \equiv\left(\rho_{\text {heavy }}-\rho_{\text {light }}\right) /\left(\rho_{\text {heavy }}+\rho_{\text {light }}\right)$. A hydrodynamic time scale is then $\tau=\lambda / v_{\mathrm{b}} \propto \sqrt{\lambda / g}$, and the hydrodynamic equations are invariant under the scale transformation $\lambda \rightarrow a_{1} \lambda, g \rightarrow a_{2} g$, and $\tau \rightarrow \sqrt{a_{1} / a_{2}} \tau[1,11]$. At $4000 \mathrm{~s}$ for the SN, the deceleration of the He-H interface is $g_{\mathrm{SN}}=-1.5 \times 10^{4}$ $\mathrm{cm} / \mathrm{s}^{2}$, the density gradient scale length is $L_{\mathrm{SN}}=\rho / \nabla \rho=8 \times 10^{10} \mathrm{~cm}$, and a characteristic perturbation wavelength is roughly $\lambda_{\mathrm{SN}} \approx 10 L_{\mathrm{SN}}=8 \times 10^{11} \mathrm{~cm}$. For the Nova experiment at $20 \mathrm{~ns} g_{\text {Nova }}=-2.5 \times 10^{13} \mathrm{~cm} / \mathrm{s}^{2}, \lambda_{\text {Nova }}=2 \times 10^{-2}$ $\mathrm{cm}$, and $\tau_{\mathrm{Nova}} \approx 5 \mathrm{~ns}$. The hydrodynamically equivalent time interval for the $\mathrm{SN}$ is then $\tau_{\mathrm{SN}}=\sqrt{a_{1} / a_{2}} \tau_{\mathrm{Nova}}=1.3 \times 10^{3} \mathrm{~s}$, a reasonable RT time scale for SN instability evolution at intermediate times. 


\section{RESULTS AND 2D SIMULATIONS}
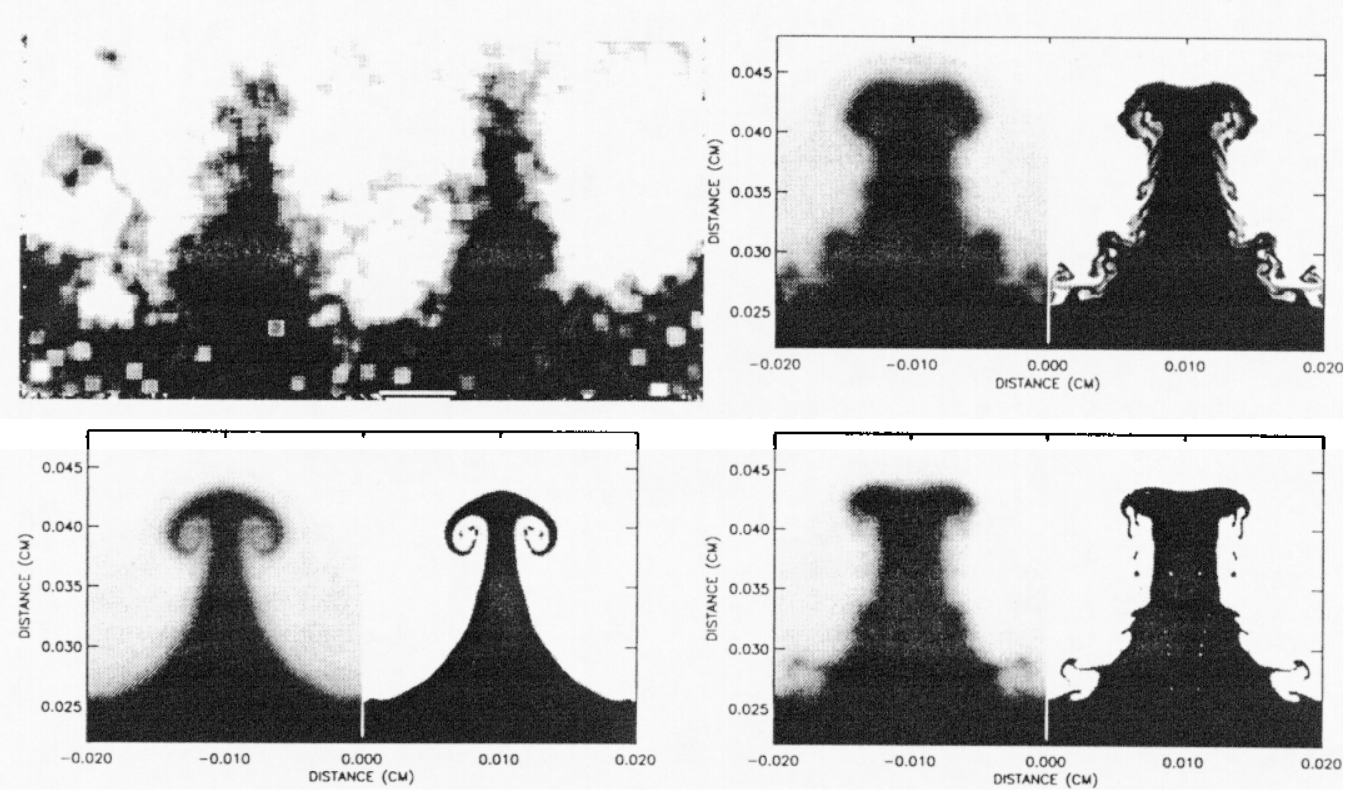

FIGURE 3. Experimental radiograph and 2D simulations at $33.2 \mathrm{~ns}$, showing the nonlinear RT growth of a $\lambda=200 \mu \mathrm{m}$ perturbation. Dark shadows of $\mathrm{Cu}$ stand out against bright regions of $\mathrm{CH}_{2}$. The left sides of the simulations have been smeared to simulate the experimental radiograph. Top left: data. Top right: PROMETHEUS, using only and ideal gas equation of state. Bottom left: CALE, ALE mode using tabular equations of state. Bottom right: CALE run the same as PROMETHEUS, in Eulerian mode with ideal gas equation of state.

Fig. 3 (top left) shows a 2D image from the experiment at $t=33.2 \mathrm{~ns}$. The other panels show results of $2 \mathrm{D}$ simulations of the experiment; in each of those other panels the right side shows the mass fraction of $\mathrm{Cu}$ with no instrument smearing, and the left side is a simulated radiograph, including the instrument resolution function of the gated x-ray framing camera $[14,28]$. In the data image, the $\mathrm{Cu}$ foil, which appears as a dark shadow, shows the classic nonlinear RT bubble-and-spike shape, and there are faint indications of a roll-up at the very tip of the spike. We initiate our 2D simulations in the same manner as in 1D: we map the conditions from the HYADES calculation at $2.45 \mathrm{~ns}$, just prior to the arrival of the shock at the thinnest part of the $\mathrm{Cu}$. The result from a CALE ALE mode simulation at $30 \mathrm{~ns}$ is shown in the bottom left panel. The initial perturbation first inverts phase (not shown) and grows due to the Richtmyer-Meshkov instability [27,23], and then continues to grow because of the RT instability. The top right panel shows the same from 2D PROMETHEUS but here using an ideal gas EOS. The gross features of the experiment are well reproduced by both simulations, however the shapes differ. The spikes are broader in PROMETHEUS, and there is more fine 
structure. When CALE is run in pure Eulerian mode with ideal gas EOS (bottom right panel), ie. in the same manner as PROMETHEUS (except for interface tracking in CALE), the codes give very similar results, but more fine structure for PROMETHEUS. The data is not yet of sufficient resolution or quality to be able to differentiate such details. In the Eulerian simulations, zones were $1 \mu \mathrm{m}$ square. Initial zoning was similar in the CALE ALE mode simulations, in that zones were $1 \mu \mathrm{m}$ wide (in the direction parallel to the interface) but the depth of zones was feathered upwards into the $\mathrm{CH}_{2}$ in the direction away from the interface (this is efficient and works very well because of the natural compression of zones in ALE mode.)

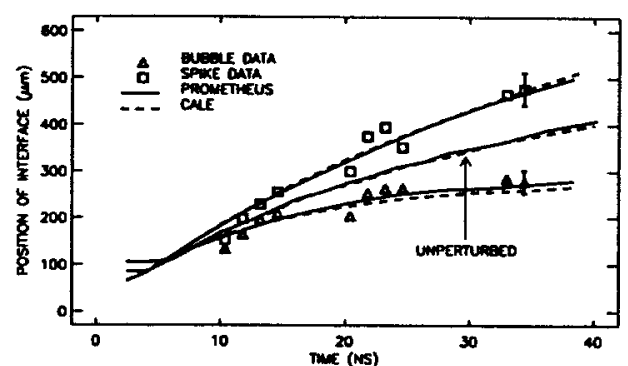

FIGURE 4. Bubble and spike position versus time. Characteristic error bars are shown for the experiment for one bubble position $( \pm 25 \mu \mathrm{m})$ and one spike position $( \pm 35 \mu \mathrm{m})$.

Fig. 4 compares spike and bubble position from the experiment, CALE, and PROMETHEUS. The observed spike and bubble fronts are well reproduced by both hydrodynamics codes. Also shown is the position of an unperturbed interface as calculated by CALE and PROMETHEUS. In the rest frame of the unperturbed interface, the bubble and spike velocities (not shown) appear to be very nearly constant, $v_{\mathrm{b}} \approx v_{\mathrm{s}}=3.5-4.0 \mu \mathrm{m} / \mathrm{ns}$. To crudely interpret this result, we use a nonlinear RT theory for incompressible semi-infinite fluids [2], which predicts that both the spike and bubble have terminal velocities given by

$$
v_{\mathrm{b}, \mathrm{s}}=\sqrt{(1 / 6 \pi)(2 A /(1 \pm A)) g \lambda} .
$$

The Atwood number is $A$, the $+(-)$ in the denominator refer to bubble (spike), and the velocities are in the frame of the unperturbed interface. Applying equation (1) at $20 \mathrm{~ns}$ gives $v_{\mathrm{b}} \approx 1.4$ and $v_{\mathrm{s}} \approx 3.1 \mu \mathrm{m} / \mathrm{ns}$. Our spike velocities are in reasonable agreement with the Alon et al. semi-infinite fluid theory, but our bubble velocities are considerably higher. The simulations show, however, that the $\mathrm{Cu}$ layer is actually a thin shell, 35-70 $\mu \mathrm{m}$ wide (see Fig. 2a), compared to the $200 \mu \mathrm{m}$ perturbation wavelength. For a low-density bubble of $\mathrm{CH}_{2}$ rising through a thin layer of $\mathrm{Cu}$, the nonlinear bubble velocity is expected to be larger than that from semi-infinite fluid theories [18], qualitatively in agreement with our simulation. We are now beginning a more careful analysis in which we account for the decompression of the materials. 


\section{2D-3D COMPARISONS}
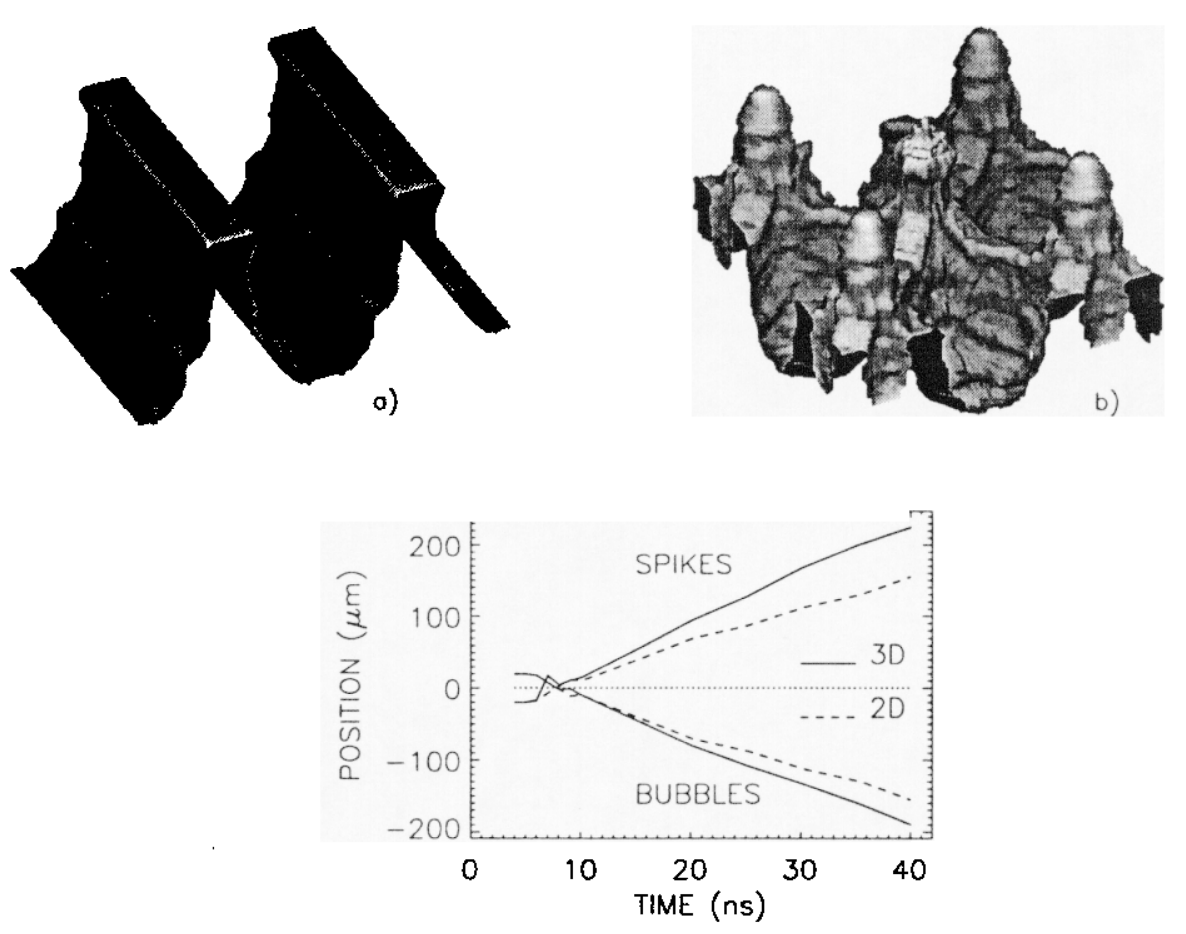

FIGURE 5. PROMETHEUS simulations of 2D vs. 3D growth in planar experiments where light foam decelerates dense CHBr. a) 2D: CHBR-foam interface at $30 \mathrm{~ns}$. Thin ridges of heavy $\mathrm{CHBr}$ penetrate between wide troughs of low density foam. Initial perturbation is $\eta_{0} * \sin \left(k_{2} y\right)$. b) 3D at 30 ns: Thin spikes penetrate between wide bubbles. Initial perturbation is $\eta_{0} * \sin \left(k_{3} y\right) \sin \left(k_{3} z\right)$, with $k_{2}=s q r t(2) * k_{3}$. Bottom panel: bubble and spike velocities in the rest frame of an unperturbed interface.

Observed velocities of ${ }^{56} \mathrm{Co}$ in SN1987A are at least $50 \%$ higher than any $2 \mathrm{D}$ simulation is able to produce $[16,13,3,15]$. Differences in growth of $2 \mathrm{D}$ and $3 \mathrm{D}$ perturbations may help explain this discrepancy. Experiments have already been done at Nova to test $2 \mathrm{D}$ vs. $3 \mathrm{D}$ growth in acceleration when the same wave number is used for both $2 \mathrm{D}$ and $3 \mathrm{D}$ [21]. We are now designing Nova experiments to test $2 \mathrm{D}$ vs. 3D growth in deceleration. Initial simulations (Fig. 5) show that 3D spike growth is $50 \%$ higher than 2D growth. The less dramatic difference in bubble growth may be due to thin-layer effects.

In conclusion, we have started experiments to benchmark aspects of the nonlinear hydrodynamics of the SN code PROMETHEUS. In the first tests, we examined the modeling of the nonlinear evolution of single-mode RT instability in 2D planar geometry. The experimental conditions are hydrodynamically similar to those at the He-H interface of a Type II SN at intermediate times $\left(10^{3}-10^{4} \mathrm{~s}\right.$.) The experimentally observed bubble and spike fronts were well reproduced by the simulations, though questions about the exact shape of the 
perturbation in the deep nonlinear regime require further investigation. We are now designing experiments to examine the difference in growth of $2 \mathrm{D}$ and $3 \mathrm{D}$ perturbations.

\section{ACKNOWLEDGMENTS}

We extend special thanks to Dave Dearborn and Bob Tipton for their help in making this project possible. We would also like to thank C. Max, D. Shvarts and U. Alon for invaluable discussions, and B. Hammel for a critical review of this manuscript.

Work performed under the auspices of the U.S. Department of Energy by the Lawrence Livermore National Laboratory under contract number W-7405ENG-48. D. A. and J. K. were supported in part by NASA grant NAGW-2450 and NSF grant ASTRO 9015976.

\section{REFERENCES}

1. Alon, U., Hecht, J., Mukamel, D., Shvarts, D., PRL 72, 2867 (1994).

2. Alon, U., Hecht, J., Ofer, D., Shvarts, D., PRL 74, 534 (1995).

3. Arnett, D., Annals N. Y. Acad. Sci. 647, 1, (1991).

4. Arnett, D., Supernovae and Nucleosynthesis, (Princeton: Princeton University Press, 1996).

5. Arnett, D., Bahcall, J. N., Kirshner, R. A., \& Woosley, S. E., Ann. Rev. A. Ap. 27, 629, (1989).

6. Arnett, D., Fryxell, B., Müller, E., ApJ 341, L63, (1989).

7. Barton, R. T., In Numerical Astrophysics, ed. J. M. Centrella, J. M. LeBlanc, R. L. Bowers, (Boston: Jones and Bartlett Publishers, Inc., 1985, pp. 482-497).

8. Blanco, V. M. et al., ApJ 320, 589 (1987).

9. Budil, K. S., et al., $P R L$ 76, 4536 (1996).

10. Budil, K. S., et al., Rev. Sci. Instrum. 67, 485 (1996).

11. Campbell, E. M., Holmes, N. C., Libby, S. B., Remington, B. A., Teller, E., submitted, Laser and Particle Beams and UCRL-JC-124258 Rev. 2 (1996).

12. Dionte, G., et al., Phys. Plasmas 3, 614 (1996).

13. Fryxell, B. A., Müller, E., Arnett, D., ApJ 367, 619 (1991).

14. Glendinning, S. G., Rev. Sci. Instrum. 63, 5108 (1992).

15. Haas, M. R. et al., ApJ 360, 257, (1990).

16. Hachisu, I., Matsuda, T., Nomoto, K., Shigeyama, T., ApJ, 390, 230 (1992).

17. Hanuschik, R. W., Dachs, J., Astron. Astrophys. 192, L29 (1987).

18. Hecht, J., Alon, U., Shvarts, D., Phys. Fluids 6, 4019 (1994).

19. Kauffman, R. L., et al., PRL 73, 2320 (1994).

20. Larsen, J. T. and Lane, S. M., J. Quant. Spect. Rad. Trans. 51, 179 (1994).

21. Marinak, M. M., Remington, B.A., et al., PRL 75, 3677 (1995).

22. McCray, R., Ann. Rev. A. Ap. 31, 175 (1993). 
23. Meshkov, E. E., Izv. Akad. Nauk SSSR Mekh. Zhidk. Gaza 4, 151, (1969). [Izv. Acad. Sci. USSR Fluid Dynamics 4, 101 (1969)]

24. Müller, E., Fryxell, B. A., Arnett, D., Astron. Astrophys. 251, 505 (1991).

25. Peyser, T. A., et al., PRL 75, 2332 (1995).

26. Lord Rayleigh, Scientific Papers II, 200, Cambridge, England (1900).

27. Richtmyer, R. D., Commun. Pure App. Math. 13, 297 (1960).

28. Robey H. F., et al., Rev. Sci. Instrum. in press (Jan. 1997).

29. Shigeyama, T., and Nomoto, K., ApJ 360, 242 (1990).

30. Taylor, Sir Geoffrey, Proc. R. Soc. London A201, 192 (1950).

31. Tueller, J., Barthelmy, S., Gehrels, N., Teegarden, B. J., Leventhal, M., MacCallum, C. J., ApJ 351, L41 (1990).

32. Witteborn, F. C., Bregman, J. D., Wooden, D. H., Pinto, P. A., Rank, D. M., Woosley, S. E., Cohen, M., ApJ 338, L9 (1989).

33. Woodward, P. R. and Colella, P., J. Comp. Phys., 54115 (1984). 


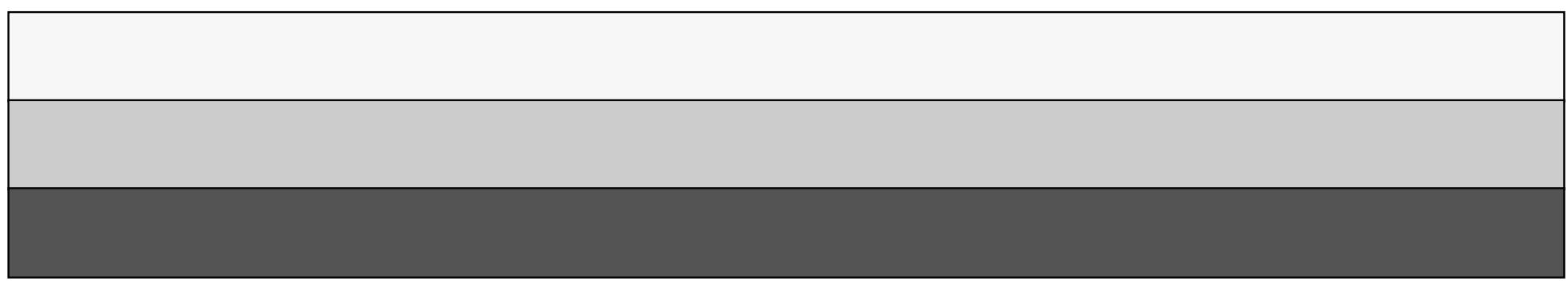

